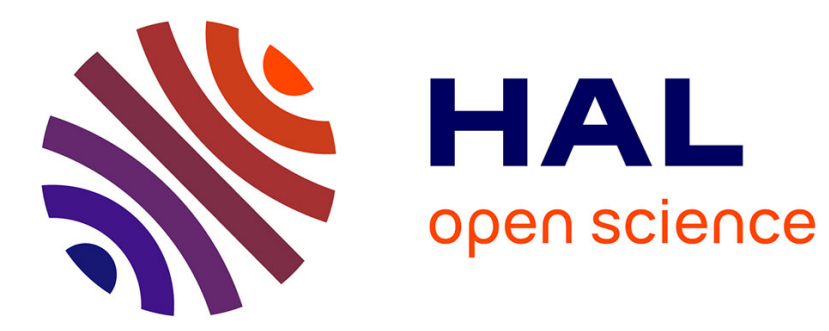

\title{
Collaboration of Cerebello-Rubral and Cerebello-Striatal Loops in a Motor Preparation Task
}

Chama Belkhiria, Eya Mssedi, Christophe N Habas, Tarak Driss, Giovanni de Marco

\section{- To cite this version:}

Chama Belkhiria, Eya Mssedi, Christophe N Habas, Tarak Driss, Giovanni de Marco. Collaboration of Cerebello-Rubral and Cerebello-Striatal Loops in a Motor Preparation Task. The Cerebellum, 2019, 18 (2), pp.203-211. 10.1007/s12311-018-0980-z . hal-02310776

\section{HAL Id: hal-02310776 \\ https://hal.parisnanterre.fr/hal-02310776}

Submitted on 10 Oct 2019

HAL is a multi-disciplinary open access archive for the deposit and dissemination of scientific research documents, whether they are published or not. The documents may come from teaching and research institutions in France or abroad, or from public or private research centers.
L'archive ouverte pluridisciplinaire HAL, est destinée au dépôt et à la diffusion de documents scientifiques de niveau recherche, publiés ou non, émanant des établissements d'enseignement et de recherche français ou étrangers, des laboratoires publics ou privés. 


\title{
Collaboration of Cerebello-Rubral and Cerebello-Striatal Loops in a Motor Preparation Task
}

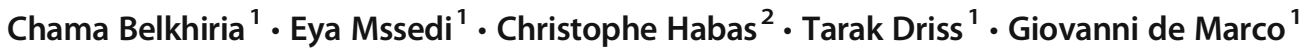

\begin{abstract}
In this study, we used fMRI to identify brain regions associated with concentration (sustained attention) during a motor preparation task. In comparison with a non-concentration task, increased activities were observed $(P<0.05$, FWEcorrected $P$ values) in cerebellar lobules VI and VII, motor cortex, pre-supplementary motor area (pre-SMA), thalamus, red nucleus (RN), and caudate nucleus $(\mathrm{CN})$. Moreover, analysis of effective connectivity inter-areal (psychophysiological interactions) showed that during preparation, concentration-related brain activity increase was dependent on Cerebellothalamo-pre-SMA-RN and Pre-SMA-CN-thalamo-M1 loops. We postulate that, while pre-SMA common to both loops is specifically involved in the movement preparation and readiness for voluntary movement through the striatum, the cerebellar lobule $\mathrm{VI}$ in conjunction with $\mathrm{RN}$, likely through a cerebellar-rubro-olivary-cerebellar loop, might be implicated in concentration-related optimization of upcoming motor performances.
\end{abstract}

Keywords Cerebellum $\cdot$ Red nucleus $\cdot$ Pre-SMA $\cdot$ Caudate nucleus $\cdot$ Motor preparation

\section{Introduction}

Motor performances are comprised of several sequential phases such as motor preparation, execution, control, and correction. Several experimental methods [1-3] have demonstrated up to $2 \mathrm{~s}$ before voluntary movement onset sequential activations in the anterior cingulate cortex [2], presupplementary motor area pre-SMA [4, 5], contralateral sensorimotor cortex, basal ganglia, and thalamus [6]. In particular, pre-SMA plays a pivotal functional role in motor preparation [7]. Pre-SMA is specifically recruited during the early phases of motor (pre) programming of overt and covert (mental preparation), complex, sequential, and externally or internally triggered movements [8]. Pre-SMA would encode spatial organization and temporal ordering of sub-movements, anticipatory postural adjustments, and inhibition of mirror

Chama Belkhiria

belkhiria.chama@gmail.com

1 Centre de Recherches sur le Sport et le Mouvement, (CeRSM - EA 2931), UFR STAPS, UPL, Université Paris Nanterre, 200 avenue de la république, 92000 Nanterre, France

2 Service de Neuro-Imagerie, Hôpital des Quinze-Vingts, UPMC Paris 6, 28, rue de Charenton, 75012 Paris, France movements [9], while pre-SMA, earlier activated, is implicated in next movement preparation, task switching/inhibition/ facilitation and motor learning.

The motor cerebellum is known to be interconnected with motor, lateral and medial premotor (SMA and pre-SMA) areas through cortico-ponto-cerebello-dentato-thalamo-cortical loops [10]. Recent studies also described anatomical cerebello-striatal connections [11]. Cerebellar lobules V-VI and VIII intervene in motor planning, execution, and online correction and learning [12], and the anterior vermis controls postural adjustments [13]. One functional hypothesis suggests that the cerebellum generates an internal model of movements via efferent copy and could compute sensory consequences of the motor command, guiding motor execution $[14,15]$. Therefore, it could be postulated that the cerebello-dentato-thalamo-pre-SMA circuit plays an important role in motor preparation, and is particularly affected during motor neuron disease. In line with this hypothesis, patients suffering from, for instance, amyotrophic lateral sclerosis, exhibited beta desynchronization between contraand ipsilateral motor cortices, increased cerebellar functional connectivity with sensorimotor areas [16], and hyperconnected subcortical motor networks between the basal ganglia and cerebellum [17]. It could be assumed that pre-SMA impairment led to either over-activation of or compensation by alternative cerebello-cortical networks. 
Furthermore, lobules VI and VII take part in the cognitive and limbic cerebellar network, i.e., executive and salience networks, and could take part in the task-related modulation of attention [18, 19]. Being a hub of (pre-) motor, limbic, and cognitive circuits, the cerebellum could monitor and synchronize different motor, limbic, and executive networks recruited by task-complexity and reward-based task. A cognitive role of the red nucleus (RN), probably in relation with salience detection and executive control, was studied in a functional magnetic resonance imaging (fMRI) investigation [20]. The resting state showed that the RN displayed strong functional coherence with associative prefrontal (pre-SMA), insular, temporal, and parietal cortices: the thalamus and the hypothalamus [20]. It has been shown [21] that functional activity of the $\mathrm{RN}$, as well as the lateral cerebellum, is primarily conducted by the sensory processing rather than by motor synchronization, suggesting that the RN may have a key role in the preparation and coordination of voluntary movements.

Previous studies indicated that the sensorimotor network is responsible for programming, executing, and controlling motor function by controlling the primary motor cortex through cerebello-thalamo-cortical connections [22]. The sensorimotor network requires the involvement of cerebellum and basal ganglia, which are functionally and anatomically interconnected. Thalamic output to motor cortex (M1) and SMA has been highlighted, and cerebellar projection to the thalamic nucleus has been mentioned. Although evidence for the role of basal ganglia in cognitive functions is increasing, they have long been associated with premotor processes [23]. Particularly, the caudate nucleus $(\mathrm{CN})$ has been shown to possess an important involvement in motor processes, planning and execution of movement, and goal-directed action [24]. The $\mathrm{CN}$ was associated with higher cognitive functions in connection with specific areas such as supplementary motor area, dorsolateral prefrontal cortex, rostral anterior cingulate, and inferior frontal gyri [24]. In a previous study [25], we showed that lobule VI was involved in the motor execution and motivation during verbal encouragement functioning as a motivational and motor integration hub.

Together, this data supports the involvement of the cortico (pre-SMA)-cerebello-thalamo-cortical pathways in motor preparation. In order to provide a more thorough understanding of the neural bases of motor preparation, we propose to model these pathways using an effective connectivity analysis. First, we sought to identify the brain regions associated with greater activity during a preparation task of concentration. Second, we show the existence of a connectivity loop formed by the right cerebellum (lobule VI), thalamus, preSMA and RN, given the crucial role that these areas are thought to play in movement preparation. We show that this loop would be influenced by a cognitive cortico-striatocortical circuit successively encompassing the pre-SMA, caudate nucleus, thalamus, and M1.

\section{Materials and Methods}

\section{Subjects}

We recruited 22 healthy, young, right-handed volunteers (11 men and 11 women, aged $30.3 \pm 4.3$ years) who gave their informed consent. The study was conducted according to the guidelines of the Declaration of Helsinki. Exclusion criteria for recruitment in the study were as follows: (i) subjects with a story of stroke or other neurological disorder, (ii) subjects with intensive practice of physical activity, (iii) other causes of significant disability like major medical or physical diseases, and (iv) major psychiatric disorders.

\section{Paradigm}

The experiment was performed during a single session and required approximately $1 \mathrm{~h}$ per participant. Inside the scanner, subjects received instructions via MR-compatible headphones. They were requested to keep their eyes closed and motionless during the whole experiment. The experimental design was comprised of a familiarization run followed by two runs. Each one of the two experimental runs included five trials. The order of each one was randomized across participants. In a run, participants mentally performed a motor preparation of $6.6 \mathrm{~s}$ and were asked to focus (concentrate) on their motor gesture before squeezing the handgrip (concentration task). In the other run, a beep prompted them to squeeze the handgrip as soon as possible; they had to squeeze the handgrip without mental preparation (non-concentration task). Both of the tasks were followed by a long rest period. The details of both tasks are presented in Fig. 1.

\section{Force Signal Measurement}

To ensure that participants performed a mental effort of concentration and increased their motivation during the preparation period, we explored the muscle force levels during the execution periods. Force signals were displayed on the computer monitor fixed in the control room. The MRI scanner (General Electric) and Biopac system (Biopac MP150, System Inc., Santa Barbara, CA) simultaneously recorded the functional brain images and force signals. Handgrip force was measured by a pressure transducer (TSD121B-MRI Hand Dynamometer). The handgrip device was an 8-m-long cable, weighed $323 \mathrm{~g}$, and was connected to the Biopac MP150. The metal-free handgrip device was held in the subject's right hand in the MRI room and was connected to the Biopac located in the MRI control room. The force signal was sampled at $200 \mathrm{~Hz}$. The output of the transducer was connected to an amplifier, DA100C, whose output was directed to AcqKnowledge software (Version 4.2, System Inc., Santa Barbara, CA). 


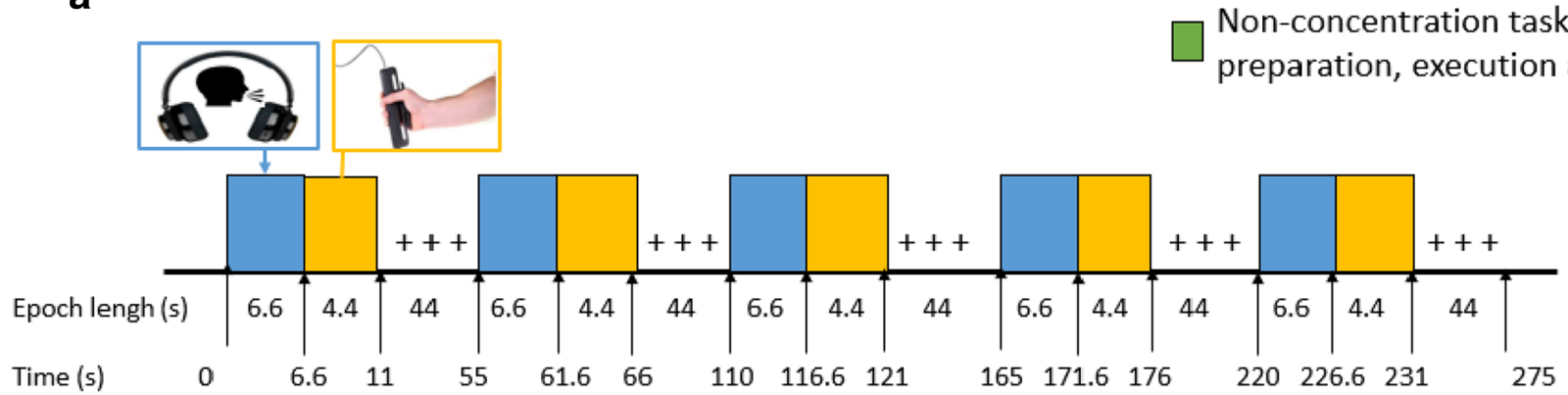

b

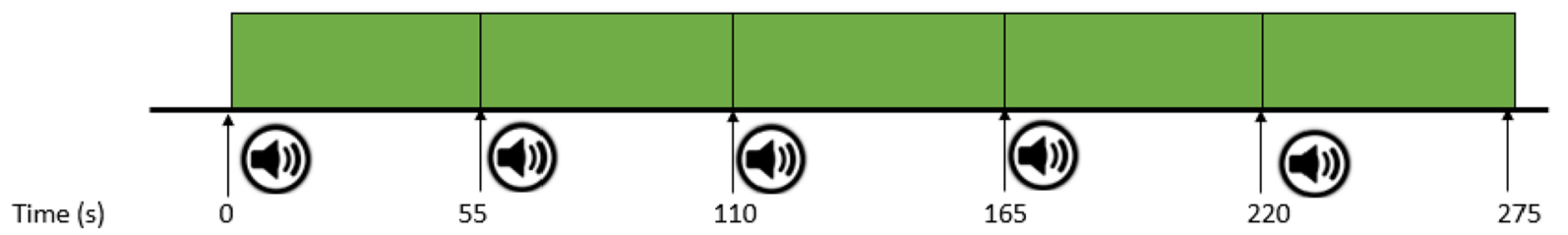

Fig. 1 Schematic timeline of the experiment. a Figure showing the concentration task where participants were verbally stimulated (instruction) to prompt concentration (blue, $6.6 \mathrm{~s}$ epochs). They were instructed not to move their hand but stay focused on their gesture. Participants were then encouraged during handgrip execution (yellow, $4.4 \mathrm{~s}$ epochs), followed by a rest period where participants relaxed ( $44 \mathrm{~s}$

\section{fMRI Data Acquisition}

A 3-T, whole-body MRI machine, equipped with a head coil (Signa; General Electric Medical System, Milwaukee, WI) was used to acquire functional $\mathrm{T} 2 *$-weighted images with blood-oxygen-level-dependent (BOLD) contrast, and highresolution anatomical T1-weighted images. For each subject, functional images consisted of $\mathrm{T} 2 *$ weighted gradient-echo echo-planar (EPI) sequence (TR/TE $=2215 / 30 \mathrm{~ms}$; flip angle $=80 ;$ matrix $=64 \times 64 ; \mathrm{FOV}=240 \times 240 \mathrm{~mm} 2$; isotropic voxel volume $=52.7 \mathrm{~mm} 3$; no interslice gap; 124 volumes $/$ run; two runs per subject). Functional images were coregistered with tridimensional anatomical images obtained with SPGR sequence (matrix, 256× 256; flip angle, 15; TR/ TE, 6.608/2.828 ms; 124 slices, 1.2-mm thickness). Head movements were restrained by cushions in order to reduce motion-related artifacts. All MR data was acquired in a single session for each subject.

\section{fMRI Image Pre-processing and Analysis}

Functional imaging data and analyses were carried out with Statistical Parametric Mapping software (SPM12, Wellcome Department of Cognitive neurology, UK, implemented in Matlab 2017, MathWorks, Natick, MA). For each subject, functional images were first corrected to adjust for withinvolume time differences, then realigned to correct for head epochs). b Diagram of the non-concentration task (green, $55 \mathrm{~s}$ epochs), including an auditory beep to signal participants to selfprepare (squeeze the handgrip without focusing on their gesture). All participants used their right hand to complete the two runs of the task. Each run included five trials

motion, co-registered with the anatomical image and smoothed in space using a three-dimensional, $8 \mathrm{~mm}$ full width at half maximum Gaussian kernel.

Hemodynamic responses to the preparation task were modeled with a canonical hemodynamic response function (HRF) and its first order temporal derivative. The motion parameters were included in the GLM as regressors to correct the movement. Anatomical masks of the regions were created using the Automated Anatomical Labeling (AAL) Atlas in the SPM Wake Forest University (WFU) PickAtlas toolbox (http:// fmri.wfubmc.edu/software/ PickAtlas, version 2.5) [26].

\section{Group Statistical Parametric Mapping}

A single-subject fixed-effect analysis was first performed followed by a random-effects analysis to make inferences to the population. An uncorrected $P$ value 0.005 for the height threshold of each activated voxel was used. While data was acquired in the whole brain, SPM was constrained to specific hypotheses concerning cerebral and cerebellar regions. A priori hypotheses allowed us to restrict statistical analyses to the M1 (-57-10 31), (pre)SMA (-9 22 52), cerebellum (lobule VI) (18 -67 -14), caudate (-18 9 20) and thalamus (-18 -16 16) regions. Results were declared significant for $P<0.05$ familywise error (FWE)-corrected for multiple comparison. 


\section{Effective Connectivity Analyses}

Effective connectivity was assessed using the method of psychophysiological interactions (PPI) [27, 28]. PPI is defined as the change in contribution from one brain area to another due to a change in experimental condition or psychological context [28]. PPI measured the connectivity between defined source and target regions. Four interactions formed the first closed connectivity loop: from lobule VI source region to thalamus to pre-SMA to RN and back to lobule VI. Three interactions formed the second connectivity loop: from preSMA source region to caudate to thalamus and then to M1. Extracted hemodynamic time series from each region of interest (seed) were deconvolved and the resulting neuronal time series (physiological variable) were combined with the cue onset times for each stimulus presented under the concentration condition (psychological variables) to derive the interaction term (source signal * experimental context). To test for differences in regression slopes between the two experimental conditions (concentration versus non-concentration), a GLM was generated with this interaction term as the explanatory variable (SPM12 Manual, chapter 36, http://www.fil.ion.ucl. ac.uk/spm/). The resulting individual t-contrast images were entered into a random-effects group analysis and tested for statistical significance at $P<0.05$ (FWE-corrected).

\section{Results}

A significant effect of concentration effort and motivation was found in the force data $(P<0.05)$ between both tasks. Indeed, the mean $( \pm \mathrm{SD})$ force values during the concentration task were $32.28 \pm 13.76 \mathrm{~kg}$ while in the non-concentration task it was $26.74 \pm 12.36 \mathrm{~kg}$. These results show that subjects improved their force overtime due to their concentration. Taking advantage of the extended motor preparation period in our paradigm, we explored the activated regions related to motor preparation and their associated connectivity.

Table 1 presents the statistical parametric results for the whole brain concerning the contrast "concentration task versus non-concentration task" during the motor preparation task. Table 1 was partitioned in the cerebellar, cortical and subcortical regions, and displays t values obtained with uncorrected $P<0.005$ values and $t$ values* obtained with FWEcorrected $P$ values $(P<0.05)$.

Brain regions of interest demonstrating significant concentration-specific changes are shown in Fig. 2. The data presents the statistical parametric maps for the regions of interests concerning the contrast "concentration task versus nonconcentration task" (FWE-corrected $P<0.05$ ) during the motor preparation task. The cerebellum (R lobule VI), left SMA, left motor cortex (M1), left thalamus, and left caudate nucleus were used as seed regions in the psychophysiological interaction analysis.

Figures 3 and 4 illustrate the psychophysiological interaction between the two connectivity loops. Figure 3 shows effective connectivity from the right cerebellar lobule VI source region to the left thalamus to the left pre-SMA target area which was connected to the left RN (FWE-corrected $P=$ 0.001).

Figure 4 shows the effective connectivity from the source region (pre-SMA) to the target region $(\mathrm{CN})$. Consistent with anatomical and functional hypotheses, the PPI analysis shows that the left (pre)SMA was significantly (FWE-corrected $P=$ 0.001) connected to the left $\mathrm{CN}$, which was significantly (FWE-corrected $P=0.003$ ) connected to the left thalamus, which was in turn was significantly connected to the left M1 (FWE-corrected $P=0.001$ ).

\section{Discussion}

The novelty of this study consists in the demonstration of a significant concentration-related increase of inter-areal effective connectivity between the cerebellar lobule VI, pre-SMA, via thalamus, and $\mathrm{RN}$. These areas may take part, at least, in a (pre-) motor cortico-cerebello-cortical loop, as well as a cerebello-rubro-olivary-cerebellar loop. We discuss below the two loops.

\section{Cerebello-Thalamo-Pre-SMA-RN Loop}

It is known that in monkeys, SMA contains force-encoding neurons [29], and ablation of SMA causes disturbed force preparation of grip [30]. In humans, Cramer et al. [31] found a strong correlation between force of squeezing and activation of SMA and S1M1. However, SMA can also play a more general role in initiating (self-paced) movement [6, 32]. Indeed, in fMRI studies, SMA has been shown to be engaged in the early phases of movement preparation, in particular, the progressive elaboration of the spatial and temporal components of the anticipatory postural adjustments (APA). A hypo-activity during gait initiation has been reported within SMA in PD patients with significantly reduced APA magnitude [33], and patients with SMA lesions exhibited an altered timing of the anticipation phase [34]. Another interesting finding concerns the rubral participation within the lobule VISMA loop. In humans, the RN receives strong projections from premotor and associative cortex [35] and from contralateral deep cerebellar nuclei, mainly from the dentate nucleus. The RN sends fibers to the bulbar olivary nucleus, through the tegmental central tract, and so takes part in a cerebello-rubroolivo-cerebellar loop modulated by cortical afferents. ICAbased fcrsMRI [35] has shown integration of the RN in the canonical motor network in humans, whereas seed-based 
Table 1 Results of activated regions in concentration task vs. non-concentration task. $B A$, Brodmann areas; MNI $(x, y, z)$ coordinates, $t$ values, uncorrected $P$ values $(P<0.005)$ and $*$ FWEcorrected $P$ values $(P<0.05)$

\begin{tabular}{|c|c|c|c|c|c|c|c|c|c|}
\hline \multirow[t]{3}{*}{ Regions } & \multirow[t]{3}{*}{$\mathrm{BA}$} & \multicolumn{3}{|c|}{ Left side } & \multirow[t]{3}{*}{$t$} & \multicolumn{3}{|c|}{ Right side } & \multirow[t]{3}{*}{$t$} \\
\hline & & \multicolumn{3}{|c|}{ MNI coordinates } & & \multicolumn{3}{|c|}{ MNI coordinates } & \\
\hline & & $x$ & $y$ & $z$ & & $x$ & $y$ & $z$ & \\
\hline \multicolumn{10}{|l|}{ Cerebellar regions } \\
\hline Lobule VI & & -15 & -76 & -14 & $3.54 *$ & 18 & -67 & -14 & $3.65^{*}$ \\
\hline Lobule VII & & -45 & -42 & -42 & 3.32 & 39 & -63 & -54 & 3.45 \\
\hline Lobule V & & -15 & -31 & -13 & 2.73 & 18 & -53 & -8 & 2.65 \\
\hline Crus I/II & & -43 & -57 & -29 & 2.11 & 39 & -67 & -23 & 2.45 \\
\hline \multicolumn{10}{|l|}{ Brainsteam } \\
\hline Red nucleus & & -9 & -22 & -2 & $3.43 *$ & 6 & -19 & -11 & $3.04 *$ \\
\hline \multicolumn{10}{|l|}{ Cortical regions } \\
\hline Suplementary motor area & 6 & -9 & 22 & 52 & $4.35^{*}$ & 9 & 13 & 55 & $3.53 *$ \\
\hline Pre-suplementary motor area & 6 & -2 & 6 & 56 & $3.58 *$ & 6 & 20 & 44 & $3.21 *$ \\
\hline Primary motor cortex & 4 & -57 & -10 & 31 & $3.52 *$ & 63 & -10 & 28 & $3.30 *$ \\
\hline Dorsolateral prefrontal cortex & 46 & -39 & 47 & 16 & 2.87 & 42 & 44 & 10 & 3.39 \\
\hline Orbitofrontal cortex & 47 & -33 & 26 & 1 & 2.55 & 21 & 32 & -8 & 2.72 \\
\hline Medial frontal gyrus & 9 & -33 & 23 & 34 & 2.22 & 30 & 32 & 37 & 3.21 \\
\hline Superior frontal gyrus & 10 & -3 & 59 & 25 & 2.81 & 36 & 35 & 10 & 3.14 \\
\hline Inferior parietal lobule & 7 & -27 & -69 & 49 & 3.16 & 27 & -58 & 46 & 2.83 \\
\hline \multicolumn{10}{|l|}{ Sub-cortical regions } \\
\hline Thalamus & & -18 & -16 & 16 & $3.63 *$ & 24 & -31 & 10 & $3.34 *$ \\
\hline Caudate & & -18 & 9 & 20 & $3.12 *$ & 14 & -14 & 26 & $2.87 *$ \\
\hline Medial globus pallidus & & -12 & -1 & -2 & 2.45 & 12 & 2 & -2 & 2.67 \\
\hline
\end{tabular}

fcrsMRI also demonstrated broader interconnections especially with associative cortices. In the current study, the effective connectivity analysis showed that RN belongs to the (pre-) motor network. In monkeys, rubral neurons showed activity related to force, speed, direction and amplitude of movements. In cats, rubral activity covaried with the amount of the rate of force change actively produced by the cat [36]. As also shown in our study, the extensive (additional) activation in the $\mathrm{RN}$ associated with concentration suggests that the RN may be involved in relaying back to the cerebellum by way of the inferior olive, information associated with cognitive behaviors. Therefore, $\mathrm{RN}$ receives movement-related inputs from the cerebellum and from the premotor cortex, likely an efferent copy during the preparation period. According to a forward model hypothesis [37], it can be supposed in our study that the rubro-olivary "couple" might act as a comparator between efferent copy and cerebellar sensory estimation of the planned movement, so as to obtain a squeeze force.

Moreover, motor preparation involves high-level cognitive processes that enable humans to anticipate action, select
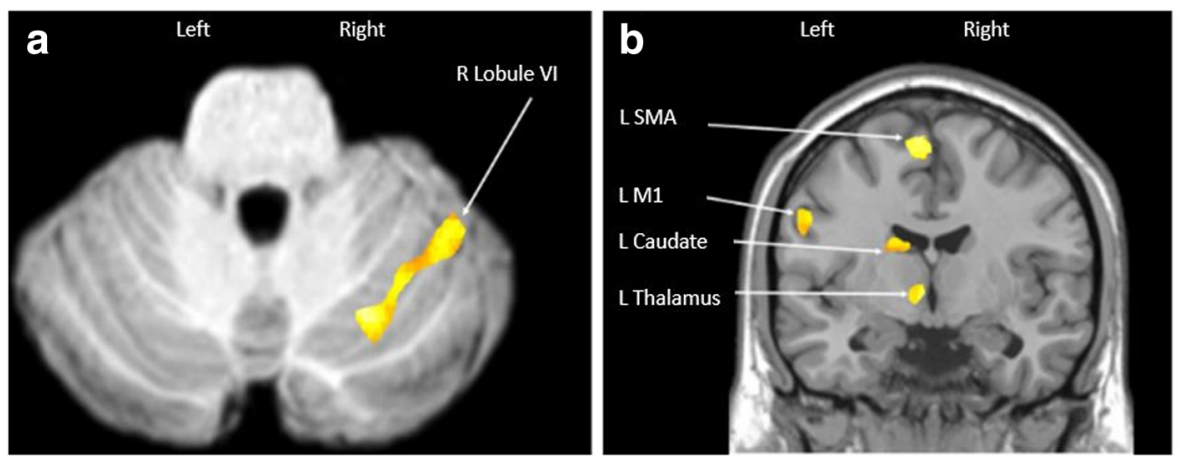

Fig. 2 Statistical parametric map of the average neural activity within the group $(N=22)(P<0.05$, corrected for multiple comparisons) during the concentration task versus non-concentration task during motor preparation. a Axial slice passing through the cerebellum and pons (lobules VI and VII). b Coronal slice passing through the encephalon in MNI space (SMA, M1, caudate, thalamus). c Axial slice passing through the dorsal red nucleus $(\mathrm{RN})$

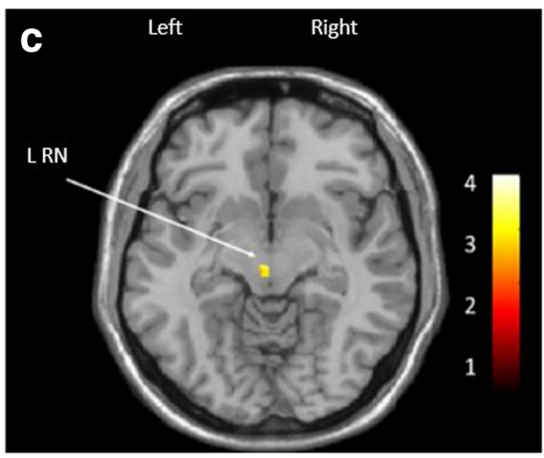




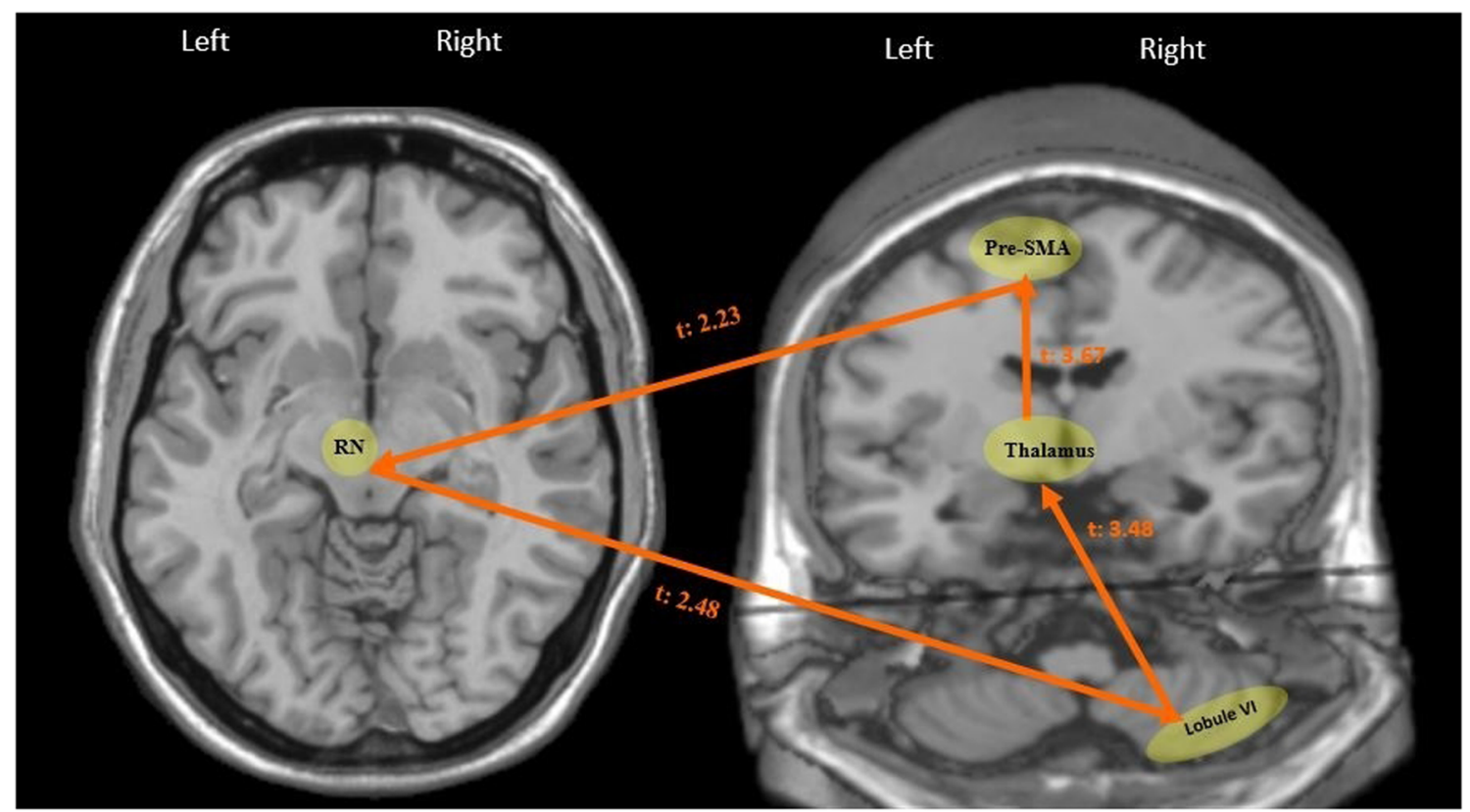

Fig. 3 Effective connectivity of the lobule VI-thalamus-pre-SMA-RN loop. The arrows reflect connectivity from source to target area: from lobule VI (20 -54 -22) to thalamus (-12 -26 8) to SMA (-2 -6 54) to RN
(-9 - 22 -2). The path coefficients reflect $t$ values of the interaction between two regions responses, decide on movement goals, and control action online [38]. The cerebellum has a crucial role in cognition; in particular, in motor control and movement planning, in the

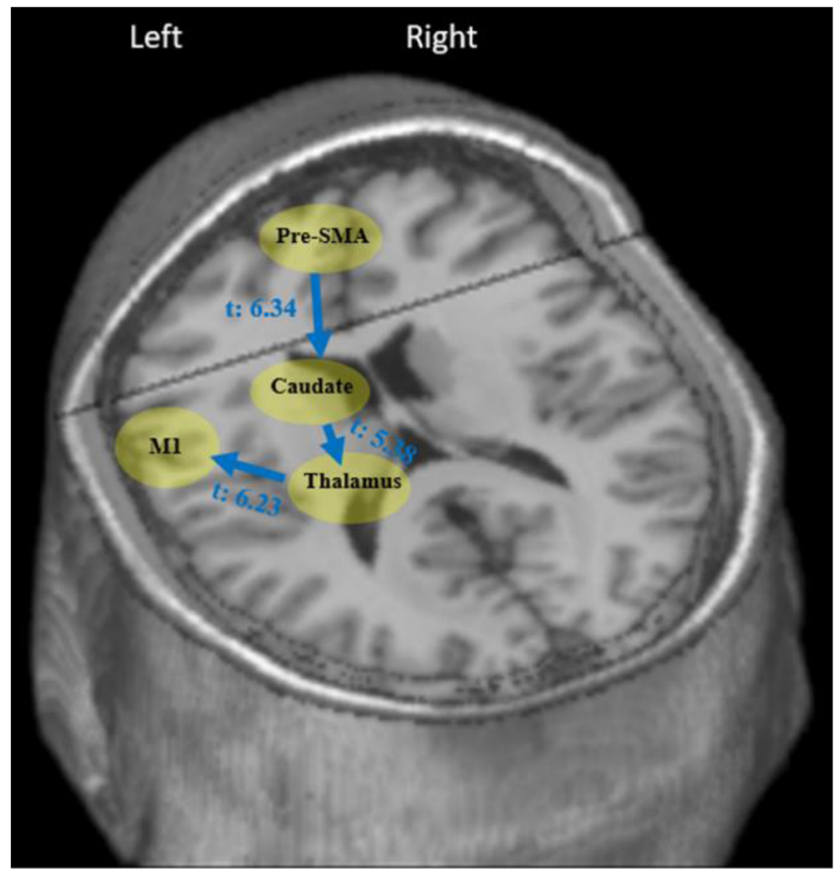

Fig. 4 Effective connectivity of the pre-SMA-caudate-thalamo-M1 circuit. The arrows reflect connectivity from source to target areas: PPI from SMA (-2 -6 54) to caudate (-10 8 4) to thalamus (-12 -26 8) and to M1 (-38 -20 62). The path coefficients reflect $t$ values of the interaction between two regions crus I/II and lobule VI. Both areas are implicated in motor control, movement planning, and posture, and are correlated with limb movement [39-41]. Also, it has been shown that the basal ganglia exhibit a preparatory activity and mediate planning and online control processes [38].In particular, the caudate nucleus specifically modulates motor planning processes that are engaged when formulating a plan of action before movement [42]. We have shown a reinforced connectivity between the RN and lobule VI during the concentrationpreparation task suggesting that the activity changes in the lobule RN are likely to be principally responsible for programming the activity changes in the lobule VI. Besides the sensorimotor cerebellum, we also recorded, at a liberal statistical threshold $(P$ value $=0.005$ uncorrected $)$, neocerebellar activations within crus I/II. These neocerebellar regions are interconnected with associative prefrontal (crus I) and parietal cortices (crus II) [18] and participate in executive networks [35]. Consequently, crus I/II could be implied in working memory (task-rule), attention/concentration to the movement preparation and initiation, and to control the internal model processing in conjunction with prefrontal areas BA 9/46, BA 9 10. It could be postulated that the cerebellum and/or the RN could constitute convergent hubs where the motor and associative cerebello-cortical loops could partially overlap and collaborate, since lobule VI is also part of the executive network [18]. It has been shown that lobules VI and VII are recruited together during the very early phase of movement learning in order to improve and automatize the motor performances [19]. The cerebellum might reflect motor commands preparation in 
order to produce the optimal motor behavior for the handgrip to be executed. This hypothesis is supported in our study by a strong connectivity between activity in the pre-SMA, RN and the lobule VI. We do not suggest that lobule VI is the sole generator of premovement activity, but rather that it facilitates the large-scale distributed cortical activity, reflected by effective connectivity, during the preparation of motor task associated with concentration. The lobule VI is positioned as a topographical hub connecting with a wide network of brain regions [25]. Its functionality is thought to be crucial to a broad range of cognitive processes such as attention and motivation [19] and executive processing [43]. In the present context of voluntary movements preparation, the lobule VI is thought to be involved in the process of action initiation. Our results advance knowledge of the crucial involvement of the lobule VI in premotor activity by showing an important contribution of the lobule VI and pre-SMA to the overall increase in cortical activity before movement.

\section{Pre-SMA-CN-Thalamo-M1 Loop}

In our study, we were able to identify significant interactions between the pre-SMA-CN, CN-thalamus and thalamus-M1. The role of primary and secondary motor areas in movement preparation has long been explored. The Bereitschaftspotential (readiness), which is measured 800 milliseconds before movement onset, was thought to originate mainly from SMA and M1 [5]. However, the contribution of these areas to motor preparation has been expanded [6]. Here, the described interactions by which the pre-SMA, CN, thalamus and M1 regulate each other's selffeedback connections therefore allow these regions to maintain sustained activity in the presence of concentration. Overall, our findings suggest that the SMA regulates the premotor activity in accordance with premovement neural activity as reflected in the two present loops. Hence, our results are in accordance with previous observations that explored the preparation of motor sequences, finding activations in the fronto-parietal regions [7], DLPFC [7], and basal ganglia [8]. Striatum (including $\mathrm{CN}$ ) is involved in planning, executing, controlling and learning/automating (skilled) movements, and in reward association learning. Therefore, the $\mathrm{CN}$ activation could reflect different intermingled functions. First, the caudate nucleus is involved in high-order processes of movement initiation, such as APA contributing to body posture, accuracy, and speed of movements [44]. CN may be implicated in motor programing by selecting, for instance, appropriate muscles (Juptner), while the cerebellum would pre-initiate dynamical parameters of the movement, using an internal model. In conjunction with pre-SMA, the CN activity may reflect an initiation and anticipation of the motion which precedes the motor execution [45]. The
pre-SMA widely projected to the basal ganglia system, which is thought to play a key role in selecting and adapting motor programs based on initial conditions by using proprioceptive input, or in formulating an internal model of body kinesthesia [23].

Likewise, some findings propose an SMA involvement in both classical and "extended" mirror neuron regions during the observation and initiation of movements [46]. The highorder information is thought to be submitted down to the basal ganglia and specifically to the striatum via fronto-striatal loops [47]. Several inputs to the basal ganglia come from the ventral striatum, which receives converging sensory input [48]. More specifically, striatal activity has been linked to action selection and control as well as the mapping of stimuli responses [49-52]. Anatomical evidence shows that the striatal projection neurons are formed by many spines on their dendrites [53], to which glutamatergic cortico-striatal axons make synaptic connection [54]. The $\mathrm{CN}$ receives spatial information via the cortico-striatal inputs and rewards related activity through the dopaminergic input [55]. Given the existence of close functional and structural connection between the striatum and the cortex, it has been shown that striatal activation was enhanced by prediction and stimulus preparation [56]. In this context, it seems possible that the $\mathrm{CN}$ associated with other cortical structures (e.g., preSMA), may be involved in anticipatory or preparatory processes during motor preparation $[57,58]$.

\section{Conclusion}

In summary, the results of this study support the existence of two distinct but collaborative loops during motor preparation: cerebello-thalamo-pre-SMA-RN and pre-SMA$C N$-thalamo-M1 loops. Lobule VI participates in the optimization of movement preparation related to the concentration and, thus, could putatively throw a bridge between motor and cognitive circuits. Lobule VI and pre-SMA may constitute hubs facilitating the motor and cognitive integration. RN may be a relay for computation of internal models that are probably coupling between the preparation and the execution phases. Further studies are needed to examine the cognitive effect on motor preparation in patients suffering from SMA or cerebellar dysfunction or attention deficit disorder. The current study gives some additional information about the red nucleus whose function in human remains matter of debate.

\section{Compliance with Ethical Standards}

Conflict of Interest The authors declare that they have no conflict of interest. 


\section{References}

1. Cunnington R, Windischberger C, Deecke L, Moser E. The preparation and readiness for voluntary movement: a high-field eventrelated fMRI study of the Bereitschafts-BOLD response. NeuroImage. 2003;20(1):404-12.

2. Nguyen VT, Breakspear M, Cunnington R. Reciprocal interactions of the SMA and cingulate cortex sustain premovement activity for voluntary actions. J Neurosci. 2014;34(49):16397-407.

3. Thickbroom G, Byrnes M, Sacco P, Ghosh S, Morris I, Mastaglia F. The role of the supplementary motor area in externally timed movement: the influence of predictability of movement timing. Brain Res. 2000;874(2):233-41.

4. Rizzolatti G, Fadiga L, Gallese V, Fogassi L. Premotor cortex and the recognition of motor actions. Brain Res Cogn Brain Res. 1996;3(2):131-41.

5. Yazawa S, Ikeda A, Kunieda T, Ohara S, Mima T, Nagamine T, et al. Human presupplementary motor area is active before voluntary movement: subdural recording of Bereitschaftspotential from medial frontal cortex. Exp Brain Res. 2000;131(2):165-77.

6. Purzner J, Paradiso GO, Cunic D, Saint-Cyr JA, Hoque T, Lozano $\mathrm{AM}$, et al. Involvement of the basal ganglia and cerebellar motor pathways in the preparation of self-initiated and externally triggered movements in humans. J Neurosci. 2007;27(22):6029-36.

7. Weilke F, Spiegel S, Boecker H, von Einsiedel HG, Conrad B, Schwaiger M, et al. Time-resolved fMRI of activation patterns in M1 and SMA during complex voluntary movement. J Neurophysiol. 2001;85(5):1858-63.

8. Lee K-M, Chang K-H, Roh J-K. Subregions within the supplementary motor area activated at different stages of movement preparation and execution. NeuroImage. 1999;9(1):117-23.

9. MacKinnon C, Bissig D, Chiusano J. Preparation of anticipatory postural adjustments prior to stepping. J Neurophysiol. 2007;97(6): 4368-79.

10. Akkal D, Dum RP, Strick PL. Supplementary motor area and presupplementary motor area: targets of basal ganglia and cerebellar output. J Neurosci. 2007;27(40):10659-73.

11. Sussman D, Leung R, Chakravarty M. The developing human brain: age-related changes in cortical, subcortical, and cerebellar anatomy. Brain Behav. 2016;6(4):e00457.

12. Rondi-Reig L, Paradis A-L, Lefort JM, Babayan BM, Tobin C. How the cerebellum may monitor sensory information for spatial representation. Front Syst Neurosci. 2014;8:205.

13. Richard A, Van Hamme A, Drevelle X, Golmard J-L, Meunier S, Welter M-L. Contribution of the supplementary motor area and the cerebellum to the anticipatory postural adjustments and execution phases of human gait initiation. Neuroscience. 2017;358:181-9.

14. D'Angelo E, Mazzarello P, Prestori F, Mapelli J, Solinas S, Lombardo P, et al. The cerebellar network: from structure to function and dynamics. Brain Res Rev. 2011;66(1-2):5-15.

15. Shadmehr R, Krakauer JW. A computational neuroanatomy for motor control. Exp Brain Res. 2008;185(3):359-81.

16. Proudfoot M, Rohenkohl G, Quinn A, Colclough GL, Wuu J, Talbot K, et al. Altered cortical beta-band oscillations reflect motor system degeneration in amyotrophic lateral sclerosis. Hum Brain Mapp. 2017;38(1):237-54.

17. Fekete T, Zach N, Mujica-Parodi L, Turner M. Multiple kernel learning captures a systems-level functional connectivity biomarker signature in amyotrophic lateral sclerosis. PLoS One. 2013;8(12): e85190.

18. Habas C, Kamdar N, Nguyen D, Prater K. Distinct cerebellar contributions to intrinsic connectivity networks. J Neurosci. 2009;29(26):8586-94.
19. Manto M, Jissendi P. Cerebellum: links between development, developmental disorders and motor learning. Front Neuroanat. 2012;6:1.

20. Nioche $\mathrm{C}$, Cabanis E. Functional connectivity of the human red nucleus in the brain resting state at 3T. AJNR Am J Neuroradiol. 2009;30(2):396-403.

21. Liu Y, Pu Y, Gao J, Parsons L, Xiong J. The human red nucleus and lateral cerebellum in supporting roles for sensory information processing. Hum Brain Mapp. 2000;10(4):147-59.

22. Penhune V, Steele C. Parallel contributions of cerebellar, striatal and M1 mechanisms to motor sequence learning. Behav Brain Res. 2012;226(2):579-91.

23. Albouy G, King B, Maquet P, Doyon J. Hippocampus and striatum: dynamics and interaction during acquisition and sleeprelated motor sequence memory consolidation. Hippocampus. 2013;23(11):985-1004.

24. Grahn J, Parkinson J, Owen A. The cognitive functions of the caudate nucleus. Prog Neurobiol. 2008;86(3):141-55.

25. Belkhiria C, Driss T, Habas C, Jaafar H, Guillevin R, de Marco G. Exploration and identification of cortico-cerebellar-brainstem closed loop during a motivational-motor task: an fMRI study. Cerebellum. 2017;16(2):326-39.

26. Tzourio-Mazoyer N, Landeau B, Papathanassiou D, Crivello F, Etard O, Delcroix N, et al. Automated anatomical labeling of activations in SPM using a macroscopic anatomical parcellation of the MNI MRI single-subject brain. NeuroImage. 2002;15(1):273-89.

27. Satoshi H, Koji J, Akira K, Osamu A, Kuni O, Yasushi M, et al. Changes in cerebro-cerebellar interaction during response inhibition after performance improvement. NeuroImage. 2014;99:142-8.

28. Stephan K, Marshall J, Friston K, Rowe J. Lateralized cognitive processes and lateralized task control in the human brain. Science. 2003;301(5631):384-6.

29. Padoa-Schioppa C, Li CSR, Bizzi E. Neuronal correlates of kinematics-to-dynamics transformation in the supplementary motor area. Neuron. 2002;36(4):751-65.

30. Smith AM, Bourbonnais D, Blanchette G. Interaction between forced grasping and a learned precision grip after ablation of the supplementary motor area. Brain Res. 1981;222(2):395-400.

31. Cramer SC, Weisskoff RM, Schaechter JD, Nelles G, Foley M, Finklestein SP, et al. Motor cortex activation is related to force of squeezing. Hum Brain Mapp. 2002;16(4):197-205.

32. Luppino G, Rizzolatti G. The organization of the frontal motor cortex. News Physiol Sci. 2000;15:219-24.

33. Kendall FP, Kendall FP. Muscles: testing and function with posture and pain: Lippincott Williams \& Wilkins; 2005.

34. Visser JE, Bloem BR. Role of the basal ganglia in balance control. Neural Plast. 2005;12(2-3):161-74.

35. Habas C, Cabanis E. Cortical projections to the human red nucleus: a diffusion tensor tractography study with a 1.5-T MRI machine. Neuroradiology. 2006;48(10):755-62.

36. Ghez C, Vicario D. The control of rapid limb movement in the cat. II. Scaling of isometric force adjustments. Exp Brain Res. 1978;33(2):191-202.

37. Ishikawa T, Tomatsu S, Izawa J, Kakei S. The cerebro-cerebellum: could it be loci of forward models? Neurosci Res. 2016;104:72-9.

38. Cerasa A, Hagberg GE, Peppe A, Bianciardi M, Gioia MC, Costa A, et al. Functional changes in the activity of cerebellum and frontostriatal regions during externally and internally timed movement in Parkinson's disease. Brain Res Bull. 2006;71(13):259-69.

39. Strick PL, Dum RP, Fiez JA. Cerebellum and nonmotor function. Annu Rev Neurosci. 2009;32(1):413-34.

40. Schmahmann JD. The role of the cerebellum in cognition and emotion: personal reflections since 1982 on the dysmetria of thought hypothesis, and its historical evolution from theory to therapy. Neuropsychol Rev. 2010;20(3):236-60. 
41. Leiner HC. Solving the mystery of the human cerebellum. Neuropsychol Rev. 2010;20(3):229-35.

42. Boecker H, Jankowski J, Ditter P, Scheef L. A role of the basal ganglia and midbrain nuclei for initiation of motor sequences. NeuroImage. 2008;39(3):1356-69.

43. Stoodley CJ, Valera EM, Schmahmann JD. Functional topography of the cerebellum for motor and cognitive tasks: an fMRI study. NeuroImage. 2012;59(2):1560-70.

44. Ng THB, Sowman PF, Brock J, Johnson BW. Neuromagnetic brain activity associated with anticipatory postural adjustments for bimanual load lifting. NeuroImage. 2013;66:343-52.

45. Bradfield LA, Balleine BW. Thalamic control of dorsomedial striatum regulates internal state to guide goal-directed action selection. J Neurosci. 2017;37(13):3721-33.

46. Sang L, Qin W, Liu Y, Han W, Zhang Y, Jiang T, et al. Resting-state functional connectivity of the vermal and hemispheric subregions of the cerebellum with both the cerebral cortical networks and subcortical structures. NeuroImage. 2012;61(4):1213-25.

47. Sege CT, Bradley MM, Lang PJ. Startle modulation during emotional anticipation and perception. Psychophysiology. 2014;51(10): 977-81.

48. Chudasama Y, Robbins TW. Functions of frontostriatal systems in cognition: comparative neuropsychopharmacological studies in rats, monkeys and humans. Biol Psychol. 2006;73(1):19-38.

49. Redgrave P, Gurney K. The short-latency dopamine signal: a role in discovering novel actions? Nat Rev Neurosci. 2006;7(12):967-75.
50. Bar-Gad I, Morris G, Bergman H. Information processing, dimensionality reduction and reinforcement learning in the basal ganglia. Prog Neurobiol. 2003;71(6):439-73.

51. Frank MJ. Computational models of motivated action selection in corticostriatal circuits. Curr Opin Neurobiol. 2011;21(3):381-6.

52. Gurney KN, Humphries M, Wood R, Prescott TJ, Redgrave P. Testing computational hypotheses of brain systems function: a case study with the basal ganglia. Network. 2004;15(4):263-90.

53. Humphries MD, Stewart RD, Gurney KN. A physiologically plausible model of action selection and oscillatory activity in the basal ganglia. J Neurosci. 2006;26(50):12921-42.

54. Kawagoe R, Takikawa Y, Hikosaka O. Expectation of reward modulates cognitive signals in the basal ganglia. Nat Neurosci. 1998;1(5):411-6.

55. Kawaguchi Y, Wilson CJ, Emson PC. Projection subtypes of rat neostriatal matrix cells revealed by intracellular injection of biocytin. J Neurosci. 1990;10(10):3421-38.

56. Smith AD, Bolam JP. The neural network of the basal ganglia as revealed by the study of synaptic connections of identified neurones. Trends Neurosci. 1990;13(7):259-65.

57. Schultz W, Apicella P, Ljungberg T, Romo R, Scarnati E. Rewardrelated activity in the monkey striatum and substantia nigra. Prog Brain Res. 1993;99:227-35.

58. Ness V, Beste C. The role of the striatum in goal activation of cascaded actions. Neuropsychologia. 2013;51(13):2562-71. 